\title{
CBED Investigations of Boron Monoarsenide Crystals
}

Yucheng Lan ${ }^{1,2}$, Bing Lv ${ }^{2,3}$, Sz-Chian Liou ${ }^{4}$, Jia-An Yan ${ }^{5}$, Jiming Bao ${ }^{6}$, Jesse Dampare ${ }^{1}$, Mobolaji Zondode $^{1}$, Rola M. Derbeshi ${ }^{1}$, Abdellah Lisfi ${ }^{1}$, Manfred R. Wuttig ${ }^{4}$, Ching-Wu Chu ${ }^{2}$, and Zhifeng Ren ${ }^{2}$

1. Department of Physics and Engineering Physics, Morgan State University, Baltimore, MD, USA

2. Department of Physics and TcSUH, University of Houston, Houston, TX, USA

3. Department of Physics, The University of Texas at Dallas, Richardson, TX, USA

4. Department of Materials Science and Engineering, University of Maryland, College Park, MD, USA

5. Department of Physics, Towson University, Baltimore, MD, USA

6. Department of Electrical and Computer Engineering, University of Houston, Houston, TX, USA

Boron monoarsenide (BAs), one III-V compound synthesized and grown since the 1960s [1-4], has been long-time ignored since its discovery. The unpopular material revokes scientists interesting recently because of its potentially high thermal conductivity [5-7], good photoelectric properties [8], and decent thermoelectric characteristic [9]. Single crystal X-ray diffraction [5] and powder X-ray diffraction [1,10] indicated that the space group of BAs should be $\mathrm{F} 3 \mathrm{~m}$ with the zinc-blende structure at room temperature, similar as other III-V arsenides and boron compounds. Here, the space group of the compound is confirmed by convergent beam electron diffraction (CBED).

BAs single crystals were grown by a two-step procedure [5]. The grown BAs crystals with a length of 100 microns were crushed and ultrasonicated in ethanol, deposited on TEM grids. The CBED was carried out using a JEM 2000FX TEM operated at $200 \mathrm{kV}$ and equipped with a $\mathrm{LaB}_{6}$ emitter. All TEM work was carried out at room temperature. Whole-pattern (WP) symmetry and bright-field (BF) symmetry of CBED patterns were taken at $15 \mathrm{~cm}$ and $150 \mathrm{~cm}$, respectively. The CBED patterns along main axes were simulated by the JEMS electron microscopy simulation software, with using the Bloch wave method at the accelerating voltage of $200 \mathrm{kV}$. The scattering factors were calculated using the Doyle and Turner atomic scattering factors for X-ray listed in International Tables for Crystallography. The electron adsorption potential was calculated by the Einstein model.

CBED patterns of BAs crystals are shown in Figures $1-3$, along $<111>,<100>$ and $<\mathrm{UWV}>$ zone axis respectively. Their symmetries are listed in Table 1. Potential diffraction groups and possible point groups are also listed in Table 1. Based on the symmetries of the CBED patterns and systematic extinction rules, the space group of BAs can be concluded as F $3 \mathrm{~m}$ (No. 216). The F $3 \mathrm{~m}$ space group was confirmed by simulations of CBED patterns. The bottom panels in Figure 1 and Figure 2 show the simulated WP and $\mathrm{BF}$ patterns along the $<111>$ and $<100>$ zone axes, respectively. The experimental CBED patterns are in good agreements with the simulated ones.

In summary, experimental and simulated CBED patterns indicated that the space group of BAs is

F 3m. The CBED conclusion is in good agreements with X-ray diffraction results and Raman scattering data [11]. 


\section{References:}

[1] J. A. Perri et al, Acta Crystallogr. 11 (1958), p. 310.

[2] S. M. Ku, J. Electrochem. Soc. 113 (1966), p. 813.

[3] A. F. Armington, J. Cryst. Growth 1 (1967), p. 47.

[4] T. L. Chu et al, J. Appl. Phys. 43 (1972), p. 276.

[5] B. Lv et al, Appl. Phys. Lett. 106 (2015), p. 074105.

[6] L. Lindsay et al, Phys. Rev. Lett. 111 (2013), p. 025901.

[7] D. A. Broido et al, Phys. Rev. B 88 (2013), p. 214303.

[8] S. Wang et al, J. Am. Chem. Soc. 134 (2012), p. 11056.

[9] J. Kim et al, Appl. Phys. Lett. 108 (2016), p. 201905.

[10] J. Osugi et al, Rev. Phys. Chem. Jpn. 36 (1966), p. 54.

[11] The work is partially supported by HDTRA122221. YCL acknowledges the support from ARL under W911NF-12-2-0022. AL is thankful to the financial support under NSF DMR 1206380 and DMR 1337916. The funding support from AFOSR (FA9550-15-1-0236) and ONR (N00014-16-1-2436) are also acknowledged.
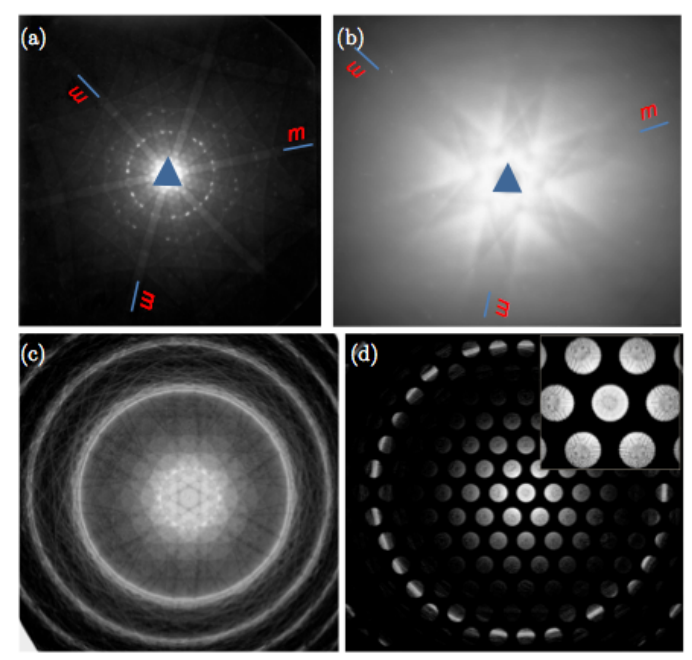

Figure 1. (a,c) WP and (b,d) BF along $<111>$ zone axis. Top: experimental; Bottom: simulated.
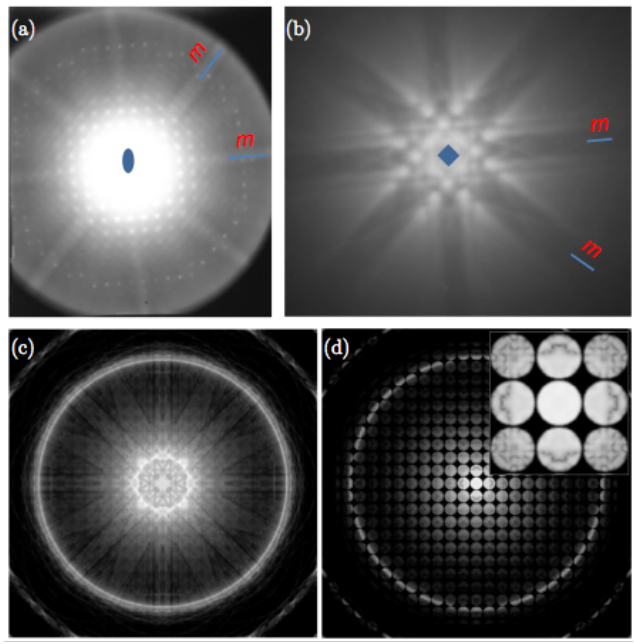

Figure 2. (a,c) WP and (b,d) BF along $<100>$ zone axis. Top: experimental; Bottom: simulated.

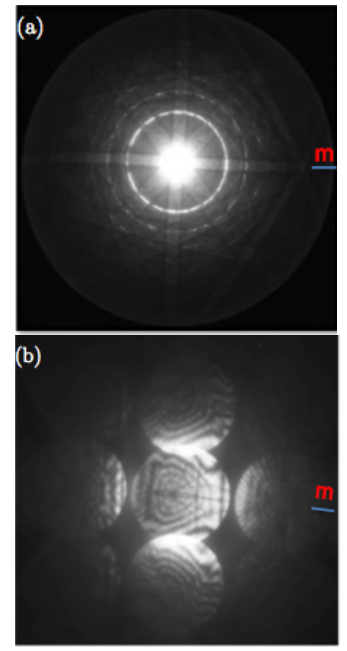

Figure 3. (a) WP and (b) BF symmetry along $<$ UVW $>$ zone axis.

TABLE I. Possible: Diffracticon Groups and Point Group

\begin{tabular}{|c|c|c|c|c|}
\hline Zone axis & WP symmetry & BF symunetry & Diffrartion groups & Possible pxint group \\
\hline$\langle 111\rangle$ & $3 \mathrm{~m}$ & $3 m$ & $\begin{array}{l}3 \mathrm{mu} \\
6 \mu \min s\end{array}$ & $\begin{array}{l}\text { 4.hn, 3n } \\
\text { masn, 3nn }\end{array}$ \\
\hline$\langle 1|x|\rangle$ & 2 man & Anm & 4 Rump & $\overline{4} 3 \mathrm{mn}, \overline{4} 2 \mathrm{~m}$ \\
\hline $\begin{array}{l}<U V W> \\
\langle U V 0>\end{array}$ & in & $\mathrm{m}$ & $\begin{array}{l}\mathrm{m} \\
2 \text { pamang }\end{array}$ & 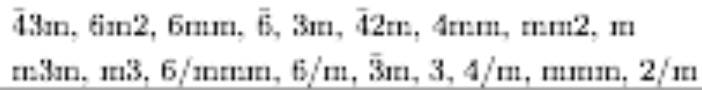 \\
\hline
\end{tabular}

